\title{
Unmanned Air Vehicle Testbed for Cooperative Control Experiments
}

Timothy W. McLain

Brigham Young University - Provo, mclain@byu.edu

Randal W. Beard

Brigham Young University - Provo, beard@byu.edu

Follow this and additional works at: https://scholarsarchive.byu.edu/facpub

Part of the Electrical and Computer Engineering Commons, and the Mechanical Engineering

Commons

\section{Original Publication Citation}

McLain, T. and Beard, R. Unmanned Air Vehicle Testbed for Cooperative Control Experiments, Proceedings of the American Control Conference, pp. 5327-5331, July 2004, Boston, Massachusetts.

\section{BYU ScholarsArchive Citation}

McLain, Timothy W. and Beard, Randal W., "Unmanned Air Vehicle Testbed for Cooperative Control Experiments" (2004). Faculty Publications. 1533.

https://scholarsarchive.byu.edu/facpub/1533

This Peer-Reviewed Article is brought to you for free and open access by BYU ScholarsArchive. It has been accepted for inclusion in Faculty Publications by an authorized administrator of BYU ScholarsArchive. For more information, please contact ellen_amatangelo@byu.edu. 


\title{
Unmanned Air Vehicle Testbed for Cooperative Control Experiments
}

\author{
Timothy W. McLain and Randal W. Beard
}

\begin{abstract}
This paper describes the MAGICC Lab unmanned air vehicle (UAV) testbed at Brigham Young University. Motivation for pursuing experimental research with UAVs is given as well as a historical perspective of the UAV testbed development. Lessons learned through the development and use of the testbed over the past several years are summarized.
\end{abstract}

\section{MOTIVATION FOR EXPERIMENTAL TESTBED}

BYU has been active in UAV research since 1999. Although our early work was simulation based, from the beginning we had an interest in testing our concepts experimentally. Our motivations were driven by our research and educational objectives.

\section{A. Research Objectives}

Our UAV research interests have centered around cooperative control of multiple vehicle systems and real-time trajectory generation [1], [2], [3]. Our primary objectives for experimental testing of our research are to validate the feasibility of practical implementation of our methods and to foster innovation to overcome implementation challenges.

For the control of UAVs, real-world issues such as sensor noise, communication dropout, communication delay, and computation latency can degrade performance and lead to catastrophic failures. Sensors that are inherently asynchronous with varied sample rates can pose challenges for estimation and coordination. Airframe payload capacity influences the choice of sensors and onboard computers and thus the inherent capabilities of the vehicle. Environmental factors, such as wind, weather, and lighting can adversely affect sensor and control system performance. Field tests often expose the unanticipated challenges that must be dealt with in a real-world scenario [4]. Furthermore, these challenges often force significant innovations to occur to enable success.

\section{B. Educational Objectives}

In the College of Engineering at BYU, the student population is roughly 80 percent undergraduate students and 20 percent graduate students. Of the graduate students, the majority are pursuing the MS degree. A major goal of the university is to provide mentored learning experiences for every undergraduate student. Involving undergraduates

T. McLain is with the Department of Mechanical Engineering, Brigham Young University, Provo, UT 84602, USA mclain@byu .edu

R. Beard is with the Department of Electrical and Computer Engineering, Brigham Young University, Provo, UT 84602, USA beard@ee.byu.edu in research is one way to accomplish this goal. One advantage of experimental research and the development of an experimental testbed is that it requires and enables a broad spectrum of engineering and research activities. These activities can draw on the talents and contributing efforts of undergraduate and graduate students alike.

UAV and robot testbed development typically demands skills from multiple disciplines including mechanisms and machine design, electronics, programming, and systems integration. Testbeds can provide opportunities for students to interact with other disciplines and often requires them to leave their comfort zones and extend beyond the scope of their classroom training.

One significant advantage of having a vehicle testbed is that it attracts student researchers. Students are anxious to gain practical experience working with hardware. Our own experience is that it is relatively easy to find students to work on research associated with a UAV testbed.

\section{BYU TESTBED EVOLUTION AND DESCRIPTIONS}

\section{A. Robot Testbeds}

The BYU Multiple AGent Intelligent Coordination and Control (MAGICC) Lab has been developing robot and UAV testbeds for educational and research purposes over the past six years. Initially, five nonholonomic robots were developed for the purposes of studying formation control and cooperative manipulation. These robots, shown in Figure 1, feature an onboard PC104 Pentium computer running the Linux operating system. They communicate with other computers over an $802.11 \mathrm{~b}$ wireless network. The position and heading of each robot is measured using an overhead vision system. More recently, these robots have been used to emulate UAVs for testing cooperative timing strategies [5]. While these tests have some benefit, the wheeled robot platform is limited in its ability to emulated fixed-wing flight due to its two-dimensional motion, relatively small field area, and significantly different sensing and communications.

Over the past two years, BYU students have developed a small omnidirectional robot platform for research in robot soccer and capture the flag games [6], [7], [8]. A closeup view of the BYU omnidirectional robot is shown in Figure 2. The omnibot is controlled by a custom controller called the MAGICC Board, which is based on the Rabbit microcontroller. One advantage of the omnibot is that it has the same computer hardware and software architecture as 


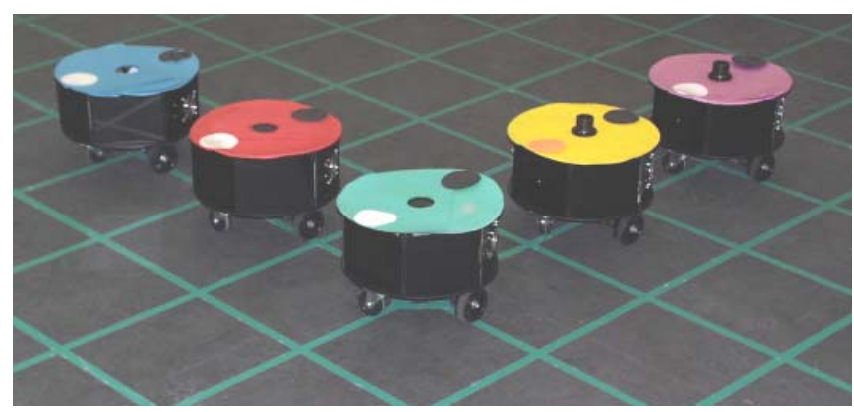

Fig. 1. MAGICC Lab nonholonomic robots

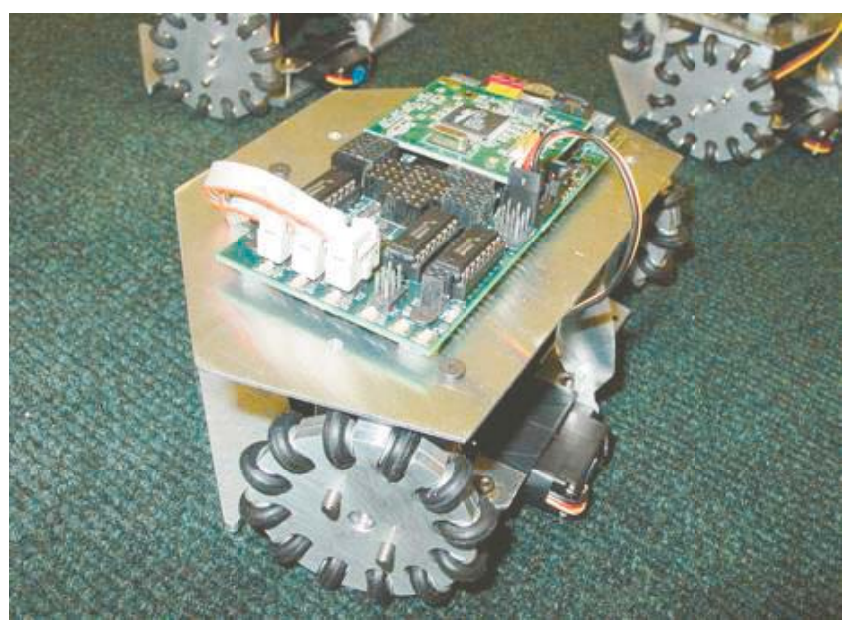

Fig. 2. MAGICC Lab omnidirectional robot

the BYU UAV autopilot, which enables sharing of software between platforms.

\section{B. UAV Testbeds}

Faculty and students in the BYU MAGICC Lab started the development of a UAV testbed about two and a half years ago. We started with an almost-ready-to-fly Model 60 Trainer aircraft with a gas engine. These planes, shown in Figure 3, feature a large $4 \mathrm{lb}$ payload and are relatively easy to assemble and fly. One disadvantage of this airframe is that it is fragile in that it cannot sustain crashes or hard landings without significant damage. This platform initially used a commercially available autopilot system [9]. Unfortunately, this autopilot failed to meet our long term research needs. It did not allow modification to the flight control software which led to difficulties in implementing the guidance and navigation capabilities required by our research.

At this point, we made two crucial decisions in our UAV platform development: First, we started building and flying EPP-foam flying-wing UAV designs. Second, we chose to modifiy the MAGICC Board for use as an autopilot for small UAVs. Photographs of several flying wing designs are shown in Figures 4 through 6. The first two UAVs are custom designs similar to the ZAGI glider that is popular with RC hobbyists. The third plane is a modified ZAGI

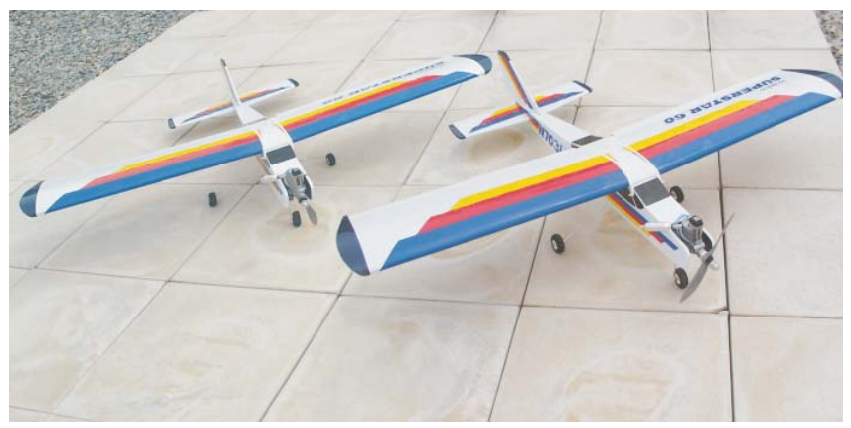

Fig. 3. MAGICC Lab Model 60 Trainer UAVs

TABLE I

UAV FLIGHT SPECIFICATIONS

\begin{tabular}{|c|c|c|c|}
\hline & MAGICC I & MAGICC II & ZAGI THL \\
\hline wingspan & $60 \mathrm{in}$ & $38 \mathrm{in}$ & $40 \mathrm{in}$ \\
payload & $32 \mathrm{oz}$ & $8 \mathrm{oz}$ & $5 \mathrm{oz}$ \\
flight time & $15 \mathrm{~min}$ & $30 \mathrm{~min}$ & $30 \mathrm{~min}$ \\
cruise speed & $30 \mathrm{mph}$ & $35 \mathrm{mph}$ & $40 \mathrm{mph}$ \\
max speed & $45 \mathrm{mph}$ & $65 \mathrm{mph}$ & $65 \mathrm{mph}$ \\
min speed & $15 \mathrm{mph}$ & $15 \mathrm{mph}$ & $15 \mathrm{mph}$ \\
\hline
\end{tabular}

THL that is available commercially [10]. The planes are constructed of EPP foam, which makes them light and durable. Each of the UAVs is battery powered and uses a DC motor in a push propeller configuration. The UAVs can be hand launched and belly landed on a grassy surface. The UAVs are controlled by elevons on the trailing edge of the wing. Fixed vertical tips on the wings provide lateral stability. Flight specifications for each of the UAVs is summarized in Table I.

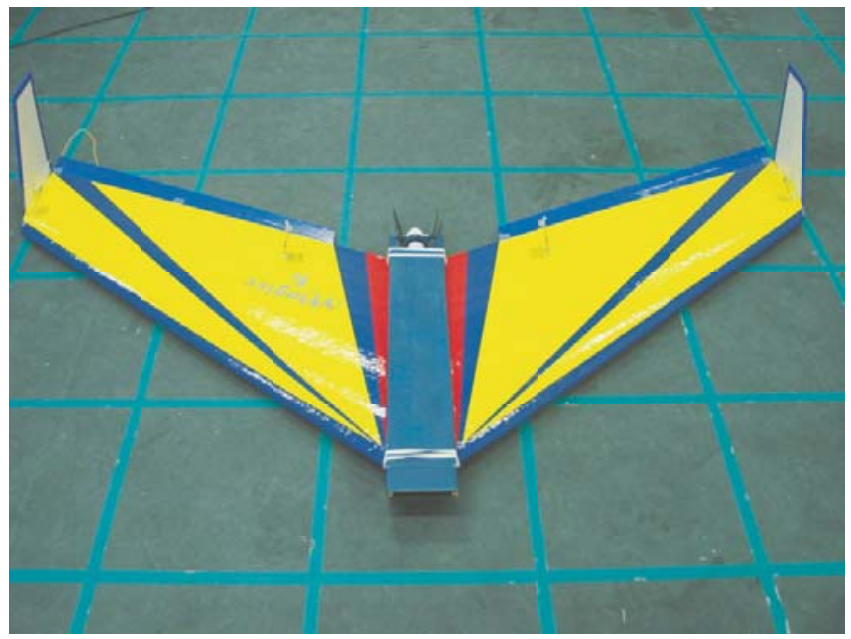

Fig. 4. MAGICC I UAV

Figure 7 shows the hardware architecture developed for our UAV systems. Key components include the autopilot which resides on the UAV and the groundstation software which runs on a laptop on the ground. Sensors on the UAV 


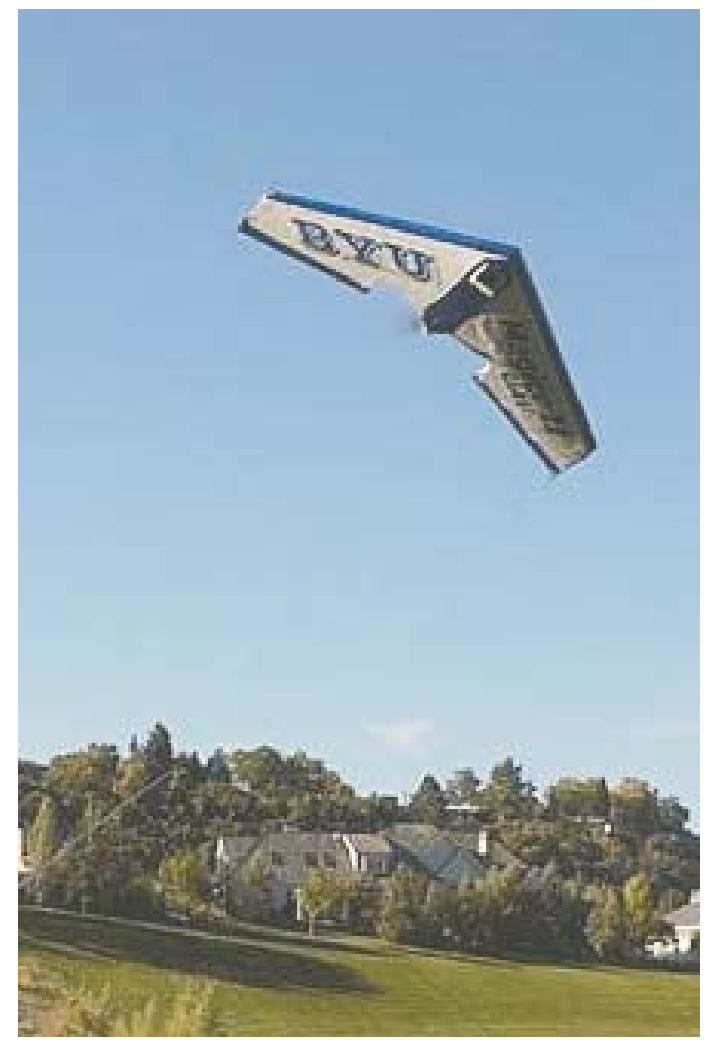

Fig. 5. MAGICC II UAV

interface with autopilot to provide critical state information. An on-board camera transmits NTSC video to the ground through a $2.4 \mathrm{GHz}$ wireless link. Video is digitized on the groundstation using frame grabber hardware. A $900 \mathrm{MHz}$ wireless modem provides a telemetry link between the groundstation laptop and the autopilot. Over this link, commands are sent from the groundstation to the autopilot. Full state and diagnostic information is sent from the UAV to the groundstation at a $5 \mathrm{~Hz}$ update rate. Operator interaction with the UAV can happen through the groundstation directly, or through PDA or voice interfaces to the groundstation. Direct RC control of the servos is enabled by a hardware by-pass switch that toggles control of the UAV between the autopilot and the RC transmitter.

All of our UAV platforms have flown using the MAGICC Lab autopilot shown in Figure 8. The CPU on the autopilot is a $29 \mathrm{MHz}$ Rabbit microcontroller with $512 \mathrm{~K}$ flash and 512K RAM. The autopilot has four servo channels, two 16-channel, 12-bit analog-to-digital converters, four serial ports, and five analog inputs. On-board sensors include three-axis rate gyros with a range of $300 \mathrm{deg} / \mathrm{s}$, threeaxis accelerometers with a range of two g's, an absolute pressure sensor capable of measuring altitude within two feet, a differential pressure sensor that can measure airspeed within $0.4 \mathrm{ft} / \mathrm{s}$, and a standard GPS receiver. The autopilot shown in Figure 8 weighs 2.3 ounces including the GPS antenna. The size of the autopilot is roughly 3.5 inches by

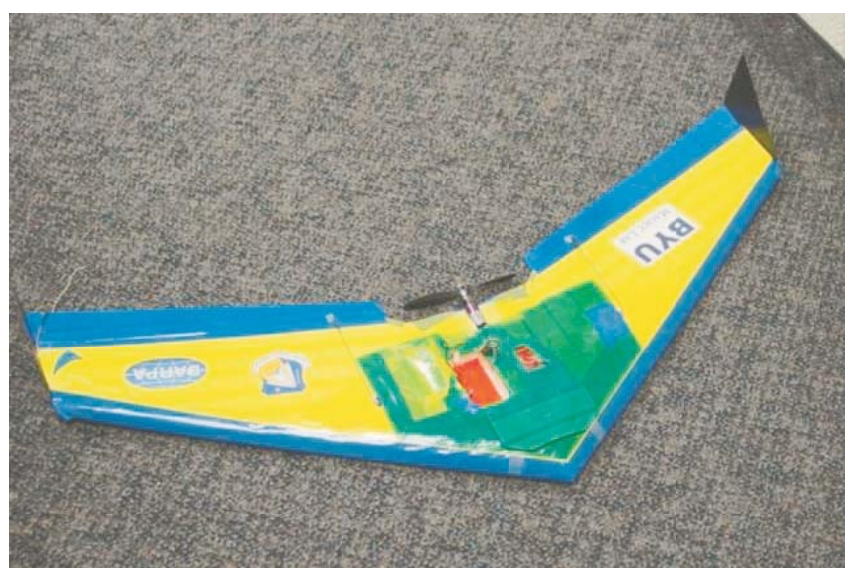

Fig. 6. Modified ZAGI UAV

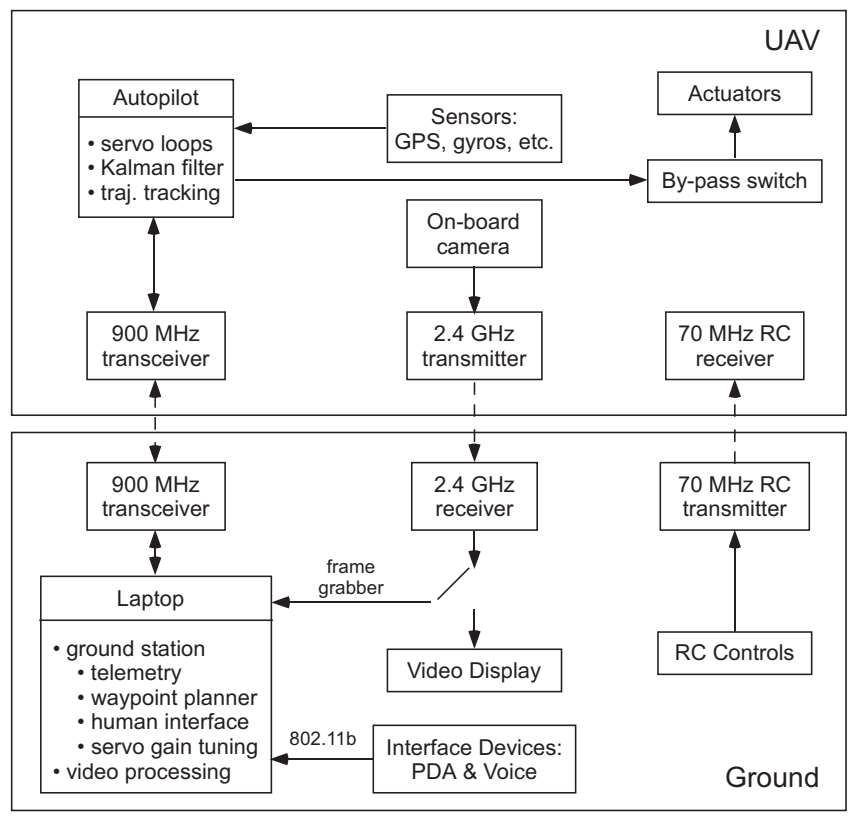

Fig. 7. System architecture

2 inches. The current revision of the autopilot is slightly smaller and weighs 1.4 ounces (without GPS antenna) with the next revision projected to weigh 0.9 ounces. Autopilot servo loops are closed at $70 \mathrm{~Hz}$ update rates.

Figure 9 shows the software architecture currently implemented onboard the MAGICC UAVs. A key feature of the current architecture is the ability to interact with the UAV at several different levels: The operator can fly the plane directly by commanding control surface deflections. At the next level, the operator can command altitude, heading, and velocity directly. At the highest level, the operator can specify a sequence of waypoints for the UAV to fly through. Operator interactions with the UAV are controlled through the Virtual Cockpit environment implemented on the groundstation laptop. A screen capture of the Virtual Cockpit is shown in Figure 10. Each of these flight modes 


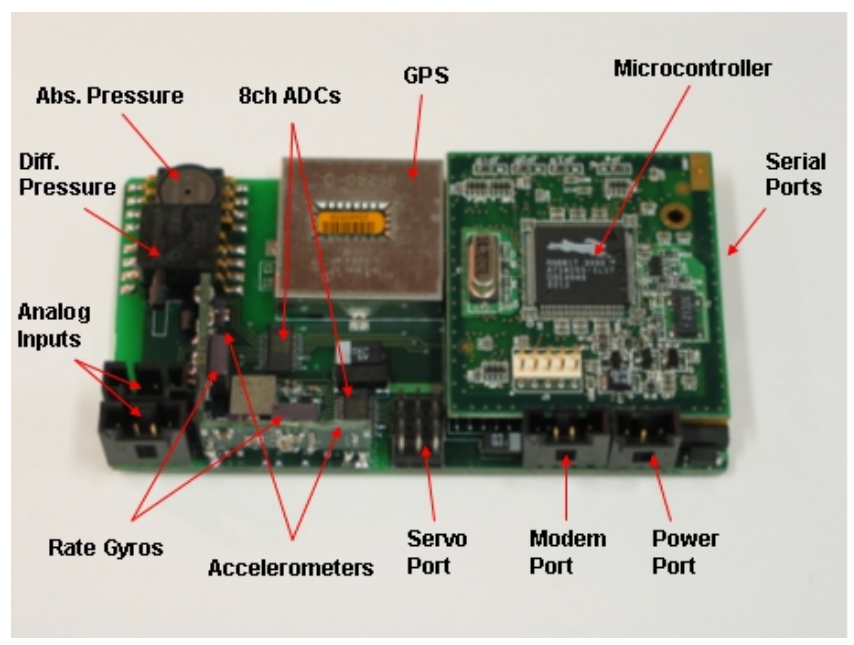

Fig. 8. MAGICC Lab autopilot hardware

has been thoroughly tested. At the present, we have completed several hundred hours of fully autonomous flight using the MAGICC UAVs, autopilot, and groundstation [11].

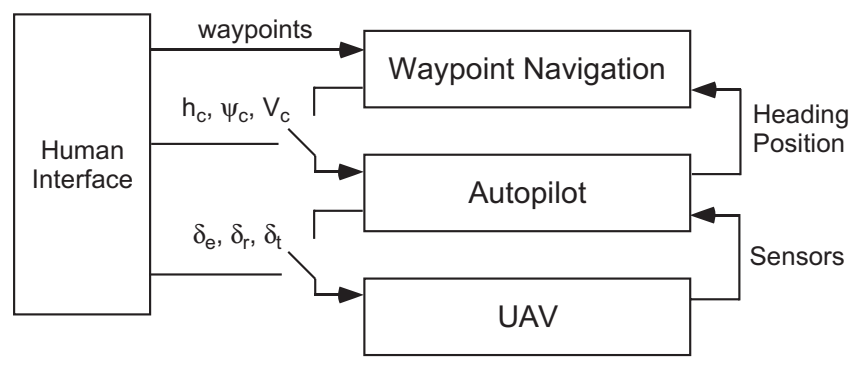

Fig. 9. Flight control software architecture

A critical extension to the Virtual Cockpit that is currently underway is to enable the control of multiple UAVs from single groundstation. Our approach involves the use of a polled communications protocol between the single wireless modem on the ground and the UAVs in the air. Using this polled approach with a single modem on the ground will slow the communications update rate significantly. Fortunately, the cooperative control strategies that we are investigating do not require high bandwidth communications between the ground and the UAVs. Commands to the UAVs will be issued at the waypoint command level. Information sent from the UAVs to the ground will be limited to diagnostic and position/attitude state information for updating the interface.

\section{THINGS WE HAVE LEARNED}

After several years of building, maintaining, and flying small UAVs, we have reached the point where we are fairly content with our setup. Some of the positive attributes of our UAV testbed include:

- Safe. Our UAVs are light weight and fly at relatively slow speeds. With their pusher props and soft foam

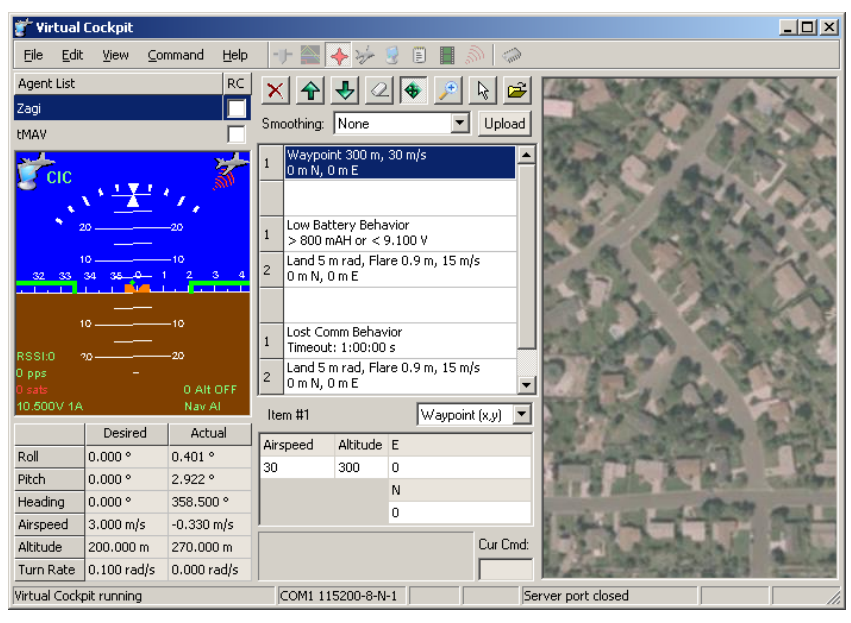

Fig. 10. Virtual Cockpit groundstation

noses, they are safe to work with and present reduced risk to general public. We feel comfortable flying at nearby public parks during hours of reduced use.

- Inexpensive. Our UAVs are relatively inexpensive to fabricate and fly. Now that autopilot development is complete, the incremental cost of producing an autopilot is much smaller than the cost of a competing product.

- Durable and Easily Repaired. In an experimental environment, the ability to sustain a crash and to recover quickly is important. The EPP foam flying wings that we fly are tough, durable, and easily repaired. The same cannot be said of the balsa and film trainers that we started with. In the case of the trainers, a crash is catastrophic and not much is left to recover.

- Fly Anywhere. Because they are relatively safe and can belly land on flat smooth terrain, our UAVs do not require an improved runway for flying. They can be launched by hand. Most of our flying takes place at a large, open city park during off-peak hours.

- Convenient Electric Power. UAVs that are powered by electric motors have a number of advantages over gas-powered planes. They are clean and do not require the storage and transport of flammable fuels. Electric motors are highly reliable and robust to variations in environmental conditions. Furthermore, they are quiet.

- Small Size. The foam flying wings that we use are small and easily transported in the smallest of automobiles.

- Accommodating Structure. The foam flying wings provide significant space for payloads provided that balance and structural integrity of the wing are not compromised. Space for additional batteries and sensor payloads can be created easily by removing foam from the wing.

- Flexible Software Architecture. Because we have developed our own autopilot, the software to control 
the UAVs can be modified at any level. We have direct control over control gains, control laws, sensor conditioning, and navigation algorithms. This capability and the capability to add new features make the MAGICC autopilot convenient for research and development activities.

The electric-powered foam flying wings do have a few negative attributes. Their payload capacity is limited. Our largest airplane ( $5 \mathrm{ft}$ wingspan) can carry about two pounds of payload. Our smallest planes only carry about half of a pound. They have limited flight duration. With a single lithium-ion battery pack, they fly for about 20 minutes. We have flown our UAVs with up to three battery packs giving an hour of flight time. These payloads and flight durations can be easily exceeded by larger gas-powered airplanes. Finally, the flying wings are not trainer airplanes and can be more difficult to fly under manual control.

\section{SUMMARY}

We have found that working with vehicle testbeds is fun and exciting for faculty and students alike. They provide great educational opportunities for students and enable valuable research and demonstrations of technology. Vehicle testbeds expose challenges that must be addressed to enable practical applications of the latest technology. Our experience is that experimental platforms can engage students intellectually and focus research efforts on fundamental problems of importance.

\section{ACKNOWLEDGMENTS}

Students have played a vital role in the development of our UAV testbed. We are grateful for the contributions of Walt Johnson, Reed Christiansen, David Hubbard, Josh Hintze, and Derek Kingston. This research has been supported by AFRL/MN under grant number F08630-03-10017, AFOSR under grant number F49620-02-C-0094, and Brigham Young University through research initiation and technology transfer grants.

\section{REFERENCES}

[1] T. McLain and R. Beard, "Cooperative rendezvous of multiple unmanned air vehicles," in Proceedings of the AIAA Guidance, Navigation and Control Conference, Denver, CO, August 2000, paper no. AIAA-2000-4369.

[2] R. W. Beard, T. W. McLain, M. Goodrich, and E. P. Anderson, "Coordinated target assignment and intercept for unmanned air vehicles," IEEE Transactions on Robotics and Automation, vol. 18, no. 6, pp. 911-922, December 2002.

[3] E. P. Anderson and R. W. Beard, "An algorithmic implementation of constrained extremal control for UAVs," in Proceedings of the AIAA Guidance, Navigation and Control Conference, Monterey, CA, August 2002, aIAA Paper No. 2002-4470.

[4] E. Hallberg, I. Kaminer, and A. Pascoal, "Development of a flight test system for unmanned air vehicles," IEEE Control Systems Magazine, vol. 19 , no. 1 , pp. 55-65, February 1999.

[5] T. W. McLain, R. W. Beard, and J. Kelsey, "Experimental demonstrations of multiple robot cooperative target intercept," in Proceedings of the AIAA Guidance, Navigation and Control Conference, Monterey, CA, August 2002, aIAA Paper No. 2002-4678.

[6] R. W. Beard, "Robot soccer: An ideal senior design experience," in Proceedings of the American Control Conference, Chicago, Illinois, June 2000, pp. 3975-3979.
[7] R. W. Beard, J. K. Archibald, and S. A. Olson, "Robot soccer as a culminating design project for undergraduates," in Proceedings of the American Control Conference, Anchorage, Alaska, May 2002.

[8] M. A. Blake, G. A. Sorensen, J. K. Archibald, and R. W. Beard, "Human assisted capture-the-flag in an urban environment," in Proceedings of the IEEE International Conference on Robotics and Automation, New Orleans, April 2004, to appear.

[9] http://www.micropilot.com/.

[10] http://zagi.com.

[11] D. Kingston, R. Beard, T. McLain, M. Larsen, and W. Ren, "Autonomous vehicle technologies for small fixed wing UAVs," in AIAA 2nd Unmanned Unlimited Systems, Technologies, and OperationsAerospace, Land, and Sea Conference and Workshop \& Exhibit, San Diego, CA, September 2003, paper no. AIAA-2003-6559. 